\title{
Applying Fuzzy AHP and Nonadditive Fuzzy Integral Methods for Evaluation and Selection of Construction Project Contractor
}

\author{
Gwo-hshiung Tzeng \\ National Chair Professor \\ Institute of Technology Management \\ National Chiao-Tung University, Taiwan \\ ghtzeng@,cc.nctu.edu.tw \\ Min-Jiu Hwang \\ Associate Professor \\ Institute of Library and Information Science \\ Hsuan-Chuang University, Taiwan \\ Jia-Horng Shieh \\ $\mathrm{Ph}$. D. Graduate \\ Institute of Technology Management \\ National Chiao-Tung University, Taiwan \\ coinbag@seed.net.tw \\ Hsin-Chi Wu \\ Master \\ Institute of Traffic and Transportation \\ National Chiao-Tung University, Taiwan
}

\begin{abstract}
keyword:fuzzy integral, fuzzy measure, fuzzy AHP, engineering management, multiple criteria decision making
\end{abstract}

Summary: The evaluation and selection of contraction works, in terms of contractor for every single category, has been made in many evaluation criteria such as cost, quality and so forth, and the conventional manner of evaluation would be: once the weight for each of the criterion would be given, which will be then summed up and ranked. However, during the processes of evaluation in practice, whether it is either weight or rate score its information would be of fuzzy problem. While on our life, there exists mutual influence among each of the evaluation criteria as well as that of several criteria is not summative, it is why this paper has chosen to employ 1 -fuzzy measure and non-additive fuzzy integral methods to replace the original aggregate summation method found in fuzzy AHP. Since the weight and rate score of conventional fuzzy integral method are of a set of definite values, it is necessary that the fuzzy weight and fuzzy rate score have to be conducted of defuzzification before the summation. In order to prevent the information in aggregate will be ranked so as to find out the best contractor. This paper will, at first, introduce fuzzy AHP, 1-measure, and the idea and method of as non-additive fuzzy integral. Then, an example will be employed to substantiate so as to correlate the disparity between non-additive fuzzy integral can better come up with such contractor with accommodate the realty even more. At the end, possible impacts on ranking will greater the $\lambda$ value and the multi-plicativeness will be higher among elements, then the difference in terms of its satisfaction (rate score) among each of the alternatives will be more pronounced.

\section{Introduction}

For the constructing or building units, construction engineering not only acquires nice planning and design but also good constructors so as to achieving the three engineering management goals with high efficiency and high quality : (1) $6 \mathrm{M}$ ( Man, Machine, Material, Money, Method, Management) within the original budget ; (2) conforming to the constructing time ; (3) the quality is better than expected to build up nice engineering hardware to satisfying the proprietors' needs. Therefore, it is key process in constructing 
engineering to choosing suitable single engineering contractor (Kerzner, 1989). However, most construct units generally take the capital into major consideration when choosing optimal contractor (let the contractors who bid lower price get the chance), regardless of the quality and management, so provoke some problems, such as rebate and jerry-build, etc; it maybe results in the civil circle and discourage the better contractors to bid. Nevertheless, it is the optimal theme for constructing and building units now that how to provide an objective and overall evaluating method to choosing proper contractors so as to improving engineering quality and satisfying the need of proprietors.

Owing to the constructing industry combines manufacturing industry and service industry, so it is more complicated than general simple and repeated manufacturing industry and its evaluating principle is more than only one and hierarchical (Kerzner, 1989); many scholars adopt AHP (Analytic Hierarchy Process)(Saaty, 1977,1980) to deal with the complicated hierarchical problems. But, during real operation, it is more easy and humanistic for interviewees to consider $\ulcorner$ principle A is much more important than principle B $\lrcorner$ than to consider $\ulcorner$ the importance of principle A and principle B is seven to one . $\lrcorner$, hence some scholars use FAHP (Fuzzy Analytic Hierarchy Process)(Buckley, 1985) to handle the obscure linguistic scale, for example, Chen and Mon(1994) applied for the option of the weapon system ; therefore, in the course of letting the interviewees express their thoughts more spontaneously and appropriately, this research adopts AHP (Fuzzy Analytic Hierarchy Process) as methodology which Buckley offered in 1985.

The basic hypothesis emphasizes the evaluation principles of the same hierarchy are respectively dependent, but in real situation it is difficult to define the problems as completely indifferent evaluation principles; that is, such as the weight of the "capital" is 0.5 , and the weight of "quality" is 0.3 , in that the two reasons influence each other ( i.e. too few capital will affect quality, and excess requirements will also affect construction time.). Therefore, the weights of "capital" and "quality" maybe consider to 0.68 instead of $0.5+0.3=0.8$. For solving the problems mentioned above, the research leads to the Fuzzy Measure and Fuzzy integral concept to deal with the problems that weights which is non-additive. The research defines it as Non-additive Fuzzy integral.

Among the present methods of calculating $\lambda$-fuzzy measure and non-additive fuzzy integral, the calculating result are all the Crisp Value, however, it is not close to the accurate situation. For instance, expressing the engineering "quality" as "great" is more realistic than expressing the measurement value of engineering "quality" is 0.75 ; similarly, the constructing time and capital are usually added or reduced for external factor (bad weather) or changing design, so it is not a crisp value but a fuzzy one. Hence, the research offers the concept about fuzzy value, $\lambda$-fuzzy measurement and non-additive fuzzy integral process; the result of calculating is a set of Fuzzy utilities, at length, we can decide the order by $\alpha$-cut.

The second section of the research will introduce fuzzy integral method, $\lambda$-fuzzy measurement, fuzzy integral, and the basic theory on the weight which is $\lambda$-fuzzy measurement and fuzzy integral; the third section will introduce the executive methods and the procedures about evaluating methods; the forth section will exemplify the evaluating methods in this research and will compare fuzzy integral method with traditional weighted, besides we will discuss how influences order when the $\lambda$ value different, and will prove the practicality; in the last section will offer conclusion and suggestion.

\section{Fuzzy AHP and Fuzzy Integral}

In order to understanding the basic theory of whole evaluating process, the section will introduce traditional AHP, fuzzy AHP, traditional $\lambda$ fuzzy measurement, and fuzzy integral method, moreover, it will introduce the $\lambda$ fuzzy measurement and fuzzy integral method which the weight is fuzzy number.

\subsection{Fuzzy Number}

\subsubsection{Triangular Fuzzy Number}


A triangular fuzzy number $\widetilde{A}$ whose value point is $\left(a_{1}, a_{2}, a_{3}\right)$ ( Fig.1), and the Membership Function will be defined as (1) :

$$
\mu_{\widetilde{A}}(x)=\left\{\begin{array}{c}
0 \quad, x<a_{1} \\
\frac{x-a_{1}}{a_{2}-a_{1}}, a_{1} \leq x \leq a_{2} \\
\frac{a_{3}-x}{a_{3}-a_{2}}, a_{2} \leq x \leq a_{3} \\
0, x>a_{3}
\end{array}\right.
$$

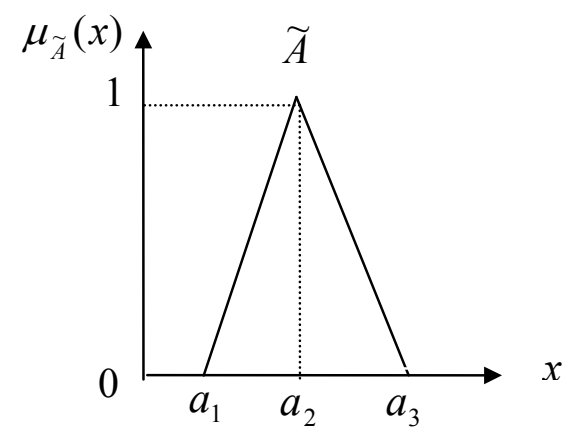

Fig1 triangular fuzzy number $\widetilde{A}$

\subsubsection{Fuzzy Number calculating}

Now there are two fuzzy numbers $\widetilde{A}=\left(a_{1}, a_{2}, a_{3}\right), \widetilde{B}=\left(b_{1}, b_{2}, b_{3}\right)$, then

$$
\begin{gathered}
\left(a_{1}, a_{2}, a_{3}\right) \oplus\left(b_{1}, b_{2}, b_{3}\right)=\left(a_{1}+b_{1}, a_{2}+b_{2}, a_{3}+b_{3}\right) \\
\left(a_{1}, a_{2}, a_{3}\right) \otimes\left(b_{1}, b_{2}, b_{3}\right)=\left(a_{1} b_{1}, a_{2} b_{2}, a_{3} b_{3}\right) \\
\widetilde{A}^{-1}=\left(a_{1}, a_{2}, a_{3}\right)^{-1} \cong\left(\frac{1}{a_{3}}, \frac{1}{a_{2}}, \frac{1}{a_{1}}\right)
\end{gathered}
$$

\subsection{3a-cut}

$$
\begin{aligned}
& \forall \alpha \in[0,1], \widetilde{A} \text { of } \alpha-c u t \text { shows }{ }^{\alpha} \widetilde{A}, \text { and } \\
& { }^{\alpha} \widetilde{A}=\left[\left(a_{2}-a_{1}\right) \alpha+a_{1},-\left(a_{3}-a_{2}\right) \alpha+a_{3}\right]=\left[{ }^{\alpha} a_{l},{ }^{\alpha} a_{r}\right]
\end{aligned}
$$

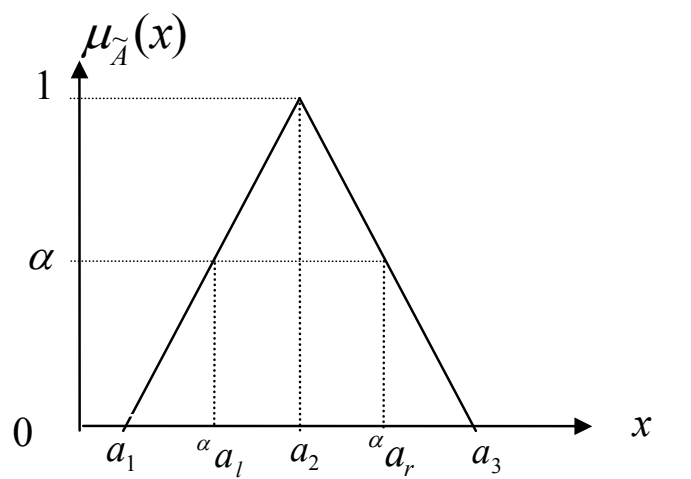


Fig.2 manner ofa-cut

\subsection{Fuzzy AHP}

\subsubsection{Traditional AHP}

Thomas L. Saaty, professor in Pittsburgh University in U.S.A, developed AHP method and it is applied popularly recently among economics, society, management field, etc. to dealing with complicated policy decision; because the limitation of the space of the page, the basic theory and methodology will not be repeated again. However, in real situation, the recognition of the interviewee is often fuzzy, thus "capital" principle "much" more important than "secure sanitary management, and If the evaluation scale which Saaty offered was expressed, the definition of $\ulcorner$ much more" maybe just 1/7, 1/8, 1/9, in other words, there exits some differences between the pair comparative values and the real recognition cognition of the interviewees. For expressing the feeling of the interviewees more accurately, the research adopts fuzzy theory to handling the linguistic scale problems.

\subsubsection{Fuzzy AHP}

Fuzzy AHP (Fuzzy Analytic hierarchy Process) was offered by Buckley in 1985; the method makes the pair comparative value in AHP offered by Saaty fuzzy, and calculates the fuzzy weight with Geometric Mean Method. The theory and methodology are as follow:

Consider a fuzzy orthogonal matrix $\widetilde{A}=\left[\widetilde{a}_{i j}\right]$, and $\widetilde{a}_{i j}=\left(\alpha_{i j}, \beta_{i j}, \gamma_{i j}, \delta_{i j}\right)$ is a trapezium fuzzy number. Taking Saaty's max- $\lambda$ method as base and considering(Tzeng and Teng, 1993)

$$
\widetilde{A} \otimes \widetilde{w}=\tilde{\lambda} \otimes \widetilde{w}
$$

in which $\widetilde{w}^{T}=\left(\widetilde{w}_{1}, \cdots, \widetilde{w}_{m}\right), \widetilde{w}_{i}=\left(\widetilde{\varepsilon}_{i}, \widetilde{\xi}_{i}, \widetilde{\eta}_{i}, \widetilde{\theta}_{i}\right), \quad \tilde{\lambda}=\left(\tilde{\lambda}_{1}, \tilde{\lambda}_{2}, \tilde{\lambda}_{3}, \tilde{\lambda}_{4}\right) \quad$ are all fuzzy numbers. $A=\left[\alpha_{i j}\right], B=\left[\beta_{i j}\right], C=\left[\gamma_{i j}\right], D=\left[\delta_{i j}\right] \quad ; \quad$ let $\quad A=\left[\alpha_{i j}\right], B=\left[\beta_{i j}\right], C=\left[\gamma_{i j}\right], D=\left[\delta_{i j}\right] \quad$; $\boldsymbol{x}^{1}=\left(\varepsilon_{1}, \cdots, \varepsilon_{m}\right)^{T}, \boldsymbol{x}^{2}=\left(\xi_{1}, \cdots, \xi_{m}\right)^{T}, \boldsymbol{x}^{3}=\left(\eta_{1}, \cdots, \eta_{m}\right)^{T}, \boldsymbol{x}^{4}=\left(\theta_{1}, \cdots, \theta_{m}\right)^{T}$

Then formula 6 will be adapted as $A x^{1}=\lambda_{1} x^{1}, B x^{2}=\lambda_{2} x^{2}, C x^{3}=\lambda_{3} x^{3}, D x^{4}=\lambda_{4} x^{4}$, Insomuch there will be four sets of max- $\lambda$ and Eigenvalue, so they can not be coped with the problem with Saaty's max- $\lambda$. Therefore Buckley led in one method for calculating fuzzy weight and fuzzy utilities.

\section{a. Fuzzy weight $\widetilde{w}_{i}$}

Hypothesizing $A=\left[a_{i j}\right]$ as a positive reciprocal matrix, and listing the geometric mean value $r_{i}=\left(\prod_{j=1}^{m} a_{i j}\right)^{1 / m}, w_{i}=r_{i} /\left(r_{1}+r_{2}+\ldots+r_{m}\right)$, If $\mathrm{m}=3$, the result is the same as Saaty's max $-\lambda$; If $\mathrm{m}>3$, the two results of both methods are pretty close.

Now we set $\tilde{A}=\left[\widetilde{a}_{i j}\right], \widetilde{a}_{i j}=\left(\alpha_{i j}, \beta_{i j}, \gamma_{i j}, \delta_{i j}\right)$ as the criteria $(1,2, \ldots, m)$ of pair comparative matrix, then the fuzzy weight of the $i$ th is

$$
\widetilde{r}_{i}=\left(\widetilde{a}_{i 1} \otimes \cdots \otimes \widetilde{a}_{i m}\right)^{1 / m}, \widetilde{w}_{i}=\widetilde{r}_{i} \otimes\left(\widetilde{r}_{1} \oplus \cdots \oplus \widetilde{r}_{m}\right)^{-1}
$$

\section{b. Fuzzy Utilities :}

If we choose the substitute program for ith principle, the fuzzy performance scores as $\widetilde{h}_{i j}$, the weight of the jth principle is $\widetilde{w}_{i}$, and the fuzzy utilities of the ith substitute program $\widetilde{u}_{i}$ is : 


$$
\tilde{u}_{i}=\sum_{j=1}^{n} \widetilde{w}_{j} \tilde{h}_{i j}
$$

\subsection{Fuzzy Measure}

In routine life, there are so many situations can not be measured with precise number (Nakajima, et al., 1994). For example, (contractor A unit price) $+($ contractor B unit) $=$ whole price, but ( the quality of contractor A $)+($ the quality of contractor B $\neq$ whole quality. Under the non-additive situation, we can lead to fuzzy measurement to meet the real require.

\subsubsection{Traditional fuzzy measurement}

The aggregation $X$ of evaluating principle consists of limited components $x_{i}, i=1,2, \ldots, n$, and shows as $X=\left\{x_{1}, x_{2}, \ldots, x_{n}\right\}, \mathrm{A}$ and $\mathrm{B}$ are defined as $\mathrm{X}$ 's part aggregation, $\mathrm{g}$ is the measurement aggregation function, $g(X)$ is g's measurement, the formulas as follow:

$$
\begin{gathered}
\quad g(\phi)=0, g(X)=1 \\
\text { when } A \subseteq B, \mathrm{~g}(A) \leq \mathrm{g}(B) \text { (simplicity) }
\end{gathered}
$$

Formula (10) also can be showed by

$$
\mathrm{g}(A \cup B) \geqq \mathrm{g}(A) \vee \mathrm{g}(B)
$$

Give a fixed $\lambda_{\text {value and make }}$

$$
g_{\lambda}(A \cup B)=g_{\lambda}(A)+g_{\lambda}(B)+\lambda g_{\lambda}(A) g_{\lambda}(B),-1<\lambda<\infty
$$

Because $g_{\lambda}($.$) meets \mathrm{g}(A \cup B) \geqq \mathrm{g}(A) \vee \mathrm{g}(B)$, Fuzzy measurement, and named $\lambda$-fuzzy measurement. Fuzzy measurement transforms into different fuzzy measurement by different $\lambda$ value. The composition is

$$
\begin{array}{lll}
\text { when } \lambda>0 & g_{\lambda}(A \cup B)>g_{\lambda}(A)+g_{\lambda}(B) & \text { multiple } \\
\text { when } \lambda=0 & g_{\lambda}(A \cup B)=g_{\lambda}(A)+g_{\lambda}(B) & \text { additive } \\
\text { when } \lambda<0 & g_{\lambda}(A \cup B)<g_{\lambda}(A)+g_{\lambda}(B) & \text { substitute }
\end{array}
$$

\subsubsection{The $\lambda$-Fuzzy measurement with fuzzy weight}

$\tilde{g}(\cdot) \cdot \tilde{0} \cdot \tilde{1}$ are fuzzy numbers $, \tilde{0}=(0,0,0), \tilde{1}=(1,1,1), X \cdot A \cdot B$ as 2.3.1 shows, then 2.3.1之formula (9)、 formula(10), formula(12)as follow :

$$
\begin{gathered}
\tilde{g}(\phi)=\widetilde{0}, \widetilde{g}(X)=\widetilde{1} \\
\text { If } A \subseteq B \text { then } \widetilde{g}(A)<\widetilde{g}(B) \\
\widetilde{g}_{\lambda}(A \cup B)=\widetilde{g}_{\lambda}(A)+\widetilde{g}_{\lambda}(B)+\lambda \widetilde{g}_{\lambda}(A) \widetilde{g}_{\lambda}(B),-1<\lambda<\infty
\end{gathered}
$$

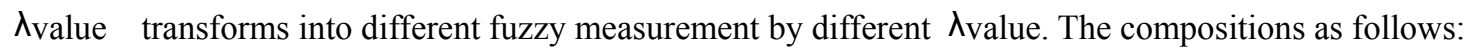

$$
\begin{array}{lll}
\text { when } \lambda>0 & \widetilde{g}_{\lambda}(A \cup B)>\widetilde{g}_{\lambda}(A)+\widetilde{g}_{\lambda}(B) & \text { multiple } \\
\text { when } \lambda=0 & \widetilde{g}_{\lambda}(A \cup B)=\widetilde{g}_{\lambda}(A)+\widetilde{g}_{\lambda}(B) & \text { additive } \\
\text { when } \lambda<0 & \widetilde{g}_{\lambda}(A \cup B)<\widetilde{g}_{\lambda}(A)+\widetilde{g}_{\lambda}(B) & \text { substitute }
\end{array}
$$




\subsection{Fuzzy Integral}

Before fuzzy integral aggregation theory emerged, researchers usually evaluate by traditional methods, say total score method and weighted mean method, etc. Take weighted mean method for example, the evaluating item are $x_{1}, x_{2}, \ldots, x_{n}$, the weight are $\widetilde{w}_{1}, \widetilde{w}_{2}, \ldots, \widetilde{w}_{n}$, and the grade of substitute program are $h_{1}=h\left(x_{1}\right), h_{2}=h\left(x_{2}\right), \ldots, h_{n}=h\left(x_{n}\right)$, according to the additive, the total utilities are:

\subsubsection{Traditional Fuzzy Integral}

$$
u_{T}=h_{1} w_{1}+h_{2} w_{2}+\ldots,+h_{n} w_{n}
$$

When weight does not confirm to the additive, comprehensive utilities are not the simple total amount, but the non-additive weighted total amount, and named fuzzy integral. The basic definition as follows:

The evaluating items are $x_{1}, x_{2}, \ldots, x_{n}$, the weights are $\widetilde{w}_{1}, \widetilde{w}_{2}, \ldots, \widetilde{w}_{n}$, and the grades of some substitute program are $h_{1}=h\left(x_{1}\right), h_{2}=h\left(x_{2}\right), \ldots, h_{n}=h\left(x_{n}\right) \quad$, suppose $h\left(x_{1}\right) \geq h\left(x_{2}\right) \geq \ldots, \geq h\left(x_{n}\right)$, then fuzzy measurement $h(\cdot)$ in the Choquet fuzzy integral of $g(\cdot)$ as follows:

$$
(C) \int h d g=h\left(x_{n}\right) g\left(H_{n}\right)+\left[h\left(x_{n-1}\right)-h\left(x_{n}\right)\right] g\left(H_{n-1}\right)+\ldots+\left[h\left(x_{1}\right)-h\left(x_{2}\right)\right] g\left(H_{1}\right)
$$

\subsubsection{The fuzzy Integral whose weight is fuzzy number}

The evaluating items are $x_{1}, x_{2}, \ldots, x_{n}$, the weights are $\widetilde{w}_{1}, \widetilde{w}_{2}, \ldots, \widetilde{w}_{n}$, and the grades of some substitute program are $h_{1}=h\left(x_{1}\right), h_{2}=h\left(x_{2}\right), \ldots, h_{n}=h\left(x_{n}\right)$, in which $\widetilde{w}_{1}, \widetilde{w}_{2}, \ldots, \widetilde{w}_{n}$ 、 $\tilde{h}_{1}, \widetilde{h}_{2}, \ldots, \tilde{h}_{n}$ are the fuzzy numbers, suppose $\tilde{h}\left(x_{1}\right) \succ \widetilde{h}\left(x_{2}\right) \succ \ldots \succ \tilde{h}\left(x_{n}\right)$ and then the fuzzy measurement of $\tilde{h}(\cdot)$ and the Chonqet fuzzy integral of $\widetilde{g}(\cdot)$ are as follow:

$$
(C) \int \tilde{h} d \widetilde{g}=\tilde{h}\left(x_{n}\right) \widetilde{g}\left(H_{n}\right)+\left[\tilde{h}\left(x_{n-1}\right)-\tilde{h}\left(x_{n}\right)\right] \widetilde{g}\left(H_{n-1}\right)+\ldots+\left[\tilde{h}\left(x_{1}\right)-\tilde{h}\left(x_{2}\right)\right] \widetilde{g}\left(H_{1}\right)
$$

\section{The establishment of evaluating and the evaluating procedure}

In order to applying for real problems, at first, this research draws up evaluating principle in light of the problems the contractors decided, besides build up one hierarchy structure for evaluation hereafter. Second, it introduces procedures and methods of Fuzzy AHP for calculating weights, so now it should obtain one set of fuzzy weight; next, it introduces each evaluating method on evaluating principle, so now it should acquire the fuzzy evaluating value of each principle and substitute program; at last, it applies the fuzzy integral method which weight is fuzzy number to add up, so it should obtain fuzzy utilities of each substitute program, then class them to acquire optimal programs.

\subsection{Evaluating principle option and building up the hierarchical structure.}

The basic definition of constructing management is that $\Gamma$ The group formed by the proprietor, the designer, and the administrator cooperates the construction and adopts professional management knowledge and technology to participating from the professional meditation stage, planning, designing, constructing to accomplishing the whole business management and that also arranges each source rationally to achieving optimal combination among constructing time, capital, and quality. $\lrcorner$ (Kerzner, 1989), therefore, the construct units invite bids or tenders not only consider the lowest price and the shortest time of the contractors but also the contractor seniority, quality of construction, and coetaneous contracting; moreover, cooperation of previous factories is also an important evaluating principle. Recently, the security and sanitary in construction site are emphasized gradually, so they not only are the important evaluating items 
but embody the image of construction unit, hence, the securely and sanitary management of previous construct site, the probability of accidents in construct sites. To combine the above concepts, the research builds a hierarchical structure as follows (see picture 3):

Fig.3 Evaluating hierarchy

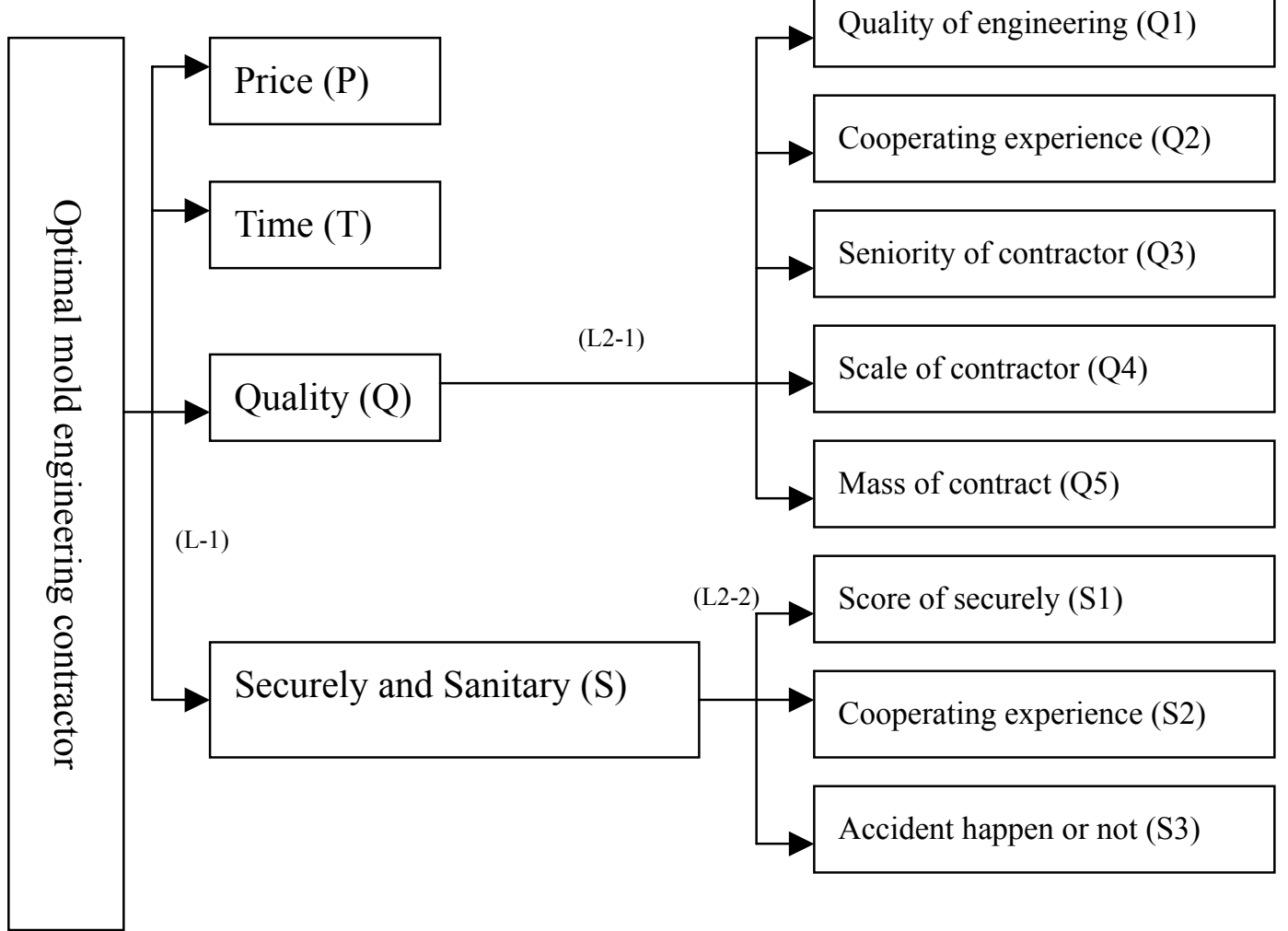

\subsection{Weight of each evaluating principle}

\subsubsection{Establish Membership Function of Linguistic Scale}

See Saaty's nine- part scale and apply for calculating of Fuzzy AHP, and the research adopts the following linguistic scales like "absolutely important", "extremely important", "very important", "a few important", "similar important", to name but a few to prescribe the relative importance between two principles.

Table 1 Membership Function of Linguistic Scale

\begin{tabular}{c|c|c}
\hline Fuzzy number & Linguistic scales & Scale of fuzzy number \\
\hline$\widetilde{1}$ & Similar important & $(1,1,3)$ \\
$\widetilde{3}$ & A few important & $(1,3,5)$ \\
$\widetilde{5}$ & Very important & $(3,5,7)$ \\
$\widetilde{7}$ & Extremely important & $(5,7,9)$ \\
$\widetilde{9}$ & Absolutely important & $(7,9,9)$ \\
\hline
\end{tabular}

\subsubsection{Establishment of pair comparative matrix}

The rating group, which consists of experienced employees and high cadres grades relative importance of each evaluating principle, and classifies into $\ulcorner$ absolutely important $\lrcorner,\ulcorner$ extremely important $\lrcorner,\ulcorner$ very important $\lrcorner,\ulcorner$ a few important $\lrcorner,\ulcorner$ similar important $\lrcorner$ five class, then endows with a relative fuzzy number as formula 19: 


$$
A^{k}=\left[\widetilde{a}_{i j}^{k}\right] \widetilde{a}_{i j}^{k}= \begin{cases}\widetilde{1}, \widetilde{3}, \widetilde{5}, \widetilde{7}, \widetilde{9}, & i<j \\ 1, & i=j \\ \widetilde{1}^{-1}, \widetilde{3}^{-1}, \widetilde{5}^{-1}, \widetilde{7}^{-1}, \widetilde{9}^{-1}, & i>j\end{cases}
$$

and in which $\widetilde{a}_{i j}^{k}=\left(a_{1}, a_{2}, a_{3}\right), \widetilde{a}_{i j}{ }^{-1}=\left(\frac{1}{a_{3}}, \frac{1}{a_{2}}, \frac{1}{a_{1}}\right)$

At last, it gains one fuzzy comparative matrix A as formula 20:

\subsection{3 calculating weight}

$$
A=\left[a_{i j}\right], a_{i j}=\left(a_{i j}^{1} \otimes a_{i j}^{2} \cdots \otimes a_{i j}^{m}\right)^{\frac{1}{m}}
$$

We can get fuzzy weight of each hierarchy by calculating from formula (7).

\subsection{Grading by each evaluating hierarchy}

\subsubsection{Establishing the membership function of linguistic scale}

The Membership Function mentioned in section 3.2.2 of the research, it adopts triangular fuzzy member to expressing linguistic scales, such as "very good", " good", "average", " bad", "very bad", and the definitions are as follows.

Table 2 Establishing the membership function of linguistic scale

\begin{tabular}{c|c|c}
\hline Fuzzy number & Linguistic scales & Scale of fuzzy number \\
\hline$\widetilde{1}$ & Very bad & $(1,1,3)$ \\
$\widetilde{3}$ & Bad & $(1,3,5)$ \\
$\widetilde{5}$ & Average & $(3,5,7)$ \\
$\widetilde{7}$ & Good & $(5,7,9)$ \\
$\widetilde{9}$ & Very good & $(7,9,9)$ \\
\hline
\end{tabular}

\subsubsection{Grading fuzzy score toward each evaluating principle}

The bid contractors provide price and finished time, and the rate group evaluates their price, time, quality, and security-sanitary, four principles, and their sub-principles; as for the quantities subscriptions about price and time are based by division of present situation about constructing site and then classified into five classes; as for quality and security-sanitary are expressed bi fixed expression, and its Fuzzy scores method as following table 3 .

After evaluating, endow with a relative fuzzy number according section 3.3.1, such as the $k$ th substitute factory, the first member of the rate group grades $\widetilde{h}_{l}{ }^{k}$ toward certain principle, suppose the group comprises m members, then the score of the first factory $\widetilde{h}_{l}$ is

$$
\widetilde{h}_{l}=\left(\widetilde{h}_{l}^{1} \otimes \widetilde{h}_{l}^{2} \cdots \otimes \widetilde{h}_{l}^{m}\right)^{\frac{1}{m}}
$$

\begin{tabular}{|c|c|c|}
\hline \multirow[t]{5}{*}{ 1.Price } & \multicolumn{2}{|c|}{$\mathrm{P}=($ bid price $)+($ single engineering budget $)$} \\
\hline & IfP $\leqq 0.9$ & "very good" class; \\
\hline & If0.9 $<\mathrm{P} \leqq 0.95$, & "good" class; \\
\hline & If $0.95<\mathrm{P} \leqq 1$ & "average" class; \\
\hline & $\begin{array}{l}\text { If } 1<\mathrm{P} \leqq 1.05 \\
\text { IfP }>1.05\end{array}$ & $\begin{array}{l}\text { "bad" class; } \\
\text { "very bad" class; }\end{array}$ \\
\hline
\end{tabular}

Table 3 Fuzzy Score Example 
Take certain constructing site for example, the construct site intents to contract some single engineering, and the budget of the engineering is $2300 \mathrm{NT}$ dollar/level ground, and predicts 12 days per floor, now there are three which named A, B, C civil engineering factories bidding. (This example simplifies the real problem, and for the privacy of the factory, it shows by its code name.) Thanks to the problem fixed to the hierarchical structure fig. 3 mentioned, so we grade the optimal contractor with the evaluating method mentioned in the third chapter. So as to collecting sufficient data about each aspect of constructing engineering as well as lest the results of evaluating turn to jaundiced, we collect a proprietor, a designer, and a manager (respectively stands for $\mathrm{E}, \mathrm{F}$ and $\mathrm{G}$ ) into a rate group to evaluating.

\subsection{Calculating each weight of hierarchy}

Building up the pair comparative matrix according to the hierarchical structure :

L-2-1

\begin{tabular}{|c|c|c|c|c|c|}
\hline \multicolumn{6}{|c|}{$\underline{\underline{E}}$ (Owners) } \\
\hline & $Q 1$ & $Q 2$ & $Q 3$ & $Q 4$ & $Q 5$ \\
\hline$\overline{Q 1}$ & 1 & $\widetilde{1}$ & $\widetilde{5}$ & $\widetilde{5}$ & $\widetilde{9}$ \\
\hline$Q 2$ & $\tilde{1}^{-1}$ & 1 & $\tilde{5}$ & $\tilde{5}$ & $\widetilde{9}$ \\
\hline$Q 3$ & $\widetilde{5}^{-1}$ & $\widetilde{5}^{-1}$ & 1 & $\tilde{1}$ & $\tilde{3}$ \\
\hline$Q 4$ & $\widetilde{5}^{-1}$ & $\widetilde{5}^{-1}$ & $\tilde{1}^{-1}$ & 1 & $\widetilde{3}$ \\
\hline$Q 5$ & $\widetilde{9}^{-1}$ & $\widetilde{9}^{-1}$ & $\widetilde{3}^{-1}$ & $\tilde{3}^{-1}$ & 1 \\
\hline
\end{tabular}

F(Designer)

\begin{tabular}{c|ccccc}
\hline & $Q 1$ & $Q 2$ & $Q 3$ & $Q 4$ & $Q 5$ \\
\hline$Q 1$ & 1 & $\widetilde{3}$ & $\widetilde{5}$ & $\widetilde{3}$ & $\widetilde{9}$ \\
$Q 2$ & $\widetilde{3}^{-1}$ & 1 & $\widetilde{3}$ & $\widetilde{5}$ & $\widetilde{7}$ \\
$Q 3$ & $\widetilde{5}^{-1}$ & $\widetilde{3}^{-1}$ & 1 & $\widetilde{1}$ & $\widetilde{5}$ \\
$Q 4$ & $\widetilde{3}^{-1}$ & $\widetilde{5}^{-1}$ & $\widetilde{1}^{-1}$ & 1 & $\widetilde{3}$ \\
$Q 5$ & $\widetilde{9}^{-1}$ & $\widetilde{7}^{-1}$ & $\widetilde{5}^{-1}$ & $\widetilde{3}^{-1}$ & 1 \\
\hline
\end{tabular}

$\underline{\mathrm{G}}($ Management)

\begin{tabular}{c|ccccc}
\hline & $Q 1$ & $Q 2$ & $Q 3$ & $Q 4$ & $Q 5$ \\
\hline$Q 1$ & 1 & $\widetilde{1}$ & $\widetilde{1}$ & $\widetilde{1}$ & $\widetilde{9}$ \\
$Q 2$ & $\widetilde{1}^{-1}$ & 1 & $\widetilde{3}$ & $\widetilde{5}$ & $\widetilde{9}$ \\
$Q 3$ & $\widetilde{1}^{-1}$ & $\widetilde{3}^{-1}$ & 1 & $\widetilde{1}$ & $\widetilde{7}$ \\
$Q 4$ & $\widetilde{1}^{-1}$ & $\widetilde{5}^{-1}$ & $\widetilde{1}^{-1}$ & 1 & $\widetilde{3}$ \\
$Q 5$ & $\widetilde{9}^{-1}$ & $\widetilde{9}^{-1}$ & $\widetilde{7}^{-1}$ & $\widetilde{3}^{-1}$ & 1 \\
\hline
\end{tabular}

E, $\mathrm{F}$ and $\mathrm{G}$ comprise a comparative matrix, and gain the mean comparative matrix by formula 20 :

\begin{tabular}{l|ccccc}
\hline & $Q 1$ & $Q 2$ & $Q 3$ & $Q 4$ & $Q 5$ \\
\hline$Q 1$ & 1 & $\left(1, \frac{13}{9}, \frac{32}{9}\right)$ & $\left(2,3, \frac{37}{7}\right)$ & $\left(\frac{13}{9}, \frac{5}{2}, \frac{33}{7}\right)$ & $(7,9,9)$ \\
$Q 2$ & $\left(\frac{9}{32}, \frac{9}{13}, 1\right)$ & 1 & $\left(\frac{13}{9}, \frac{5}{2}, \frac{28}{5}\right)$ & $(3,5,7)$ & $\left(\frac{28}{5}, 7,9\right)$ \\
$Q 3$ & $\left(\frac{71}{37}, \frac{1}{3}, \frac{1}{2}\right)$ & $\left(\frac{5}{28}, \frac{2}{5}, \frac{9}{13}\right)$ & 1 & $(1,1,3)$ & $\left(\frac{5}{2}, \frac{33}{7}, \frac{34}{5}\right)$ \\
$Q 4$ & $\left(\frac{7}{33}, \frac{2}{5}, \frac{9}{13}\right)$ & $\left(\frac{1}{7}, \frac{1}{5}, \frac{1}{3}\right)$ & $\left(\frac{1}{3}, 1,1\right)$ & 1 & $(1,3,5)$ \\
$Q 5$ & $\left(\frac{1}{9}, \frac{1}{9}, \frac{1}{7}\right)$ & $\left(\frac{1}{9}, \frac{1}{7}, \frac{5}{28}\right)$ & $\left(\frac{5}{34}, \frac{7}{33}, \frac{2}{5}\right)$ & $\left(\frac{1}{5}, \frac{1}{3}, 1\right)$ & 1 \\
\hline
\end{tabular}

According to mean comparative matrix to calculating the fuzzy weight by formula 7:

$(\mathrm{Q} 1, \mathrm{Q} 2, \mathrm{Q} 3, \mathrm{Q} 4, \mathrm{Q} 5)=[(1.46,1.88,3.13),(1.15,1.77,2.58),(0.47,0.69,1.13),(0.31,0.58,0.82),(0.16$, $0.16,0.31)]$

L-2-2 after equalizing (owing to the limitation of the paper space, just list the mean comparative matrix)

\begin{tabular}{c|ccc}
\hline & $S 1$ & $S 2$ & $S 3$ \\
\hline$S 1$ & 1 & $\left(\frac{13}{9}, \frac{5}{2}, \frac{33}{7}\right)$ & $\left(\frac{5}{2}, \frac{13}{4}, \frac{23}{4}\right)$ \\
$S 2$ & $\left(\frac{7}{33}, \frac{2}{5}, \frac{9}{13}\right)$ & 1 & $(1,3,5)$ \\
$S 3$ & $\left(\frac{4}{23}, \frac{4}{13}, \frac{2}{5}\right)$ & $\left(\frac{1}{5}, \frac{1}{3}, 1\right)$ & 1 \\
\hline
\end{tabular}

Calculating fuzzy weight by formula 7 :

$(\mathrm{S} 1, \mathrm{~S} 2, \mathrm{~S} 3)=[(1.31,1.74,2.73),(0.51,0.93,1.38),(0.30,0.41,0.66)]$

L-1 after equalizing 


\begin{tabular}{c|cccc}
\hline & $P$ & $T$ & $Q$ & $S$ \\
\hline$P$ & 1 & $\left(\frac{5}{2}, \frac{33}{7}, \frac{34}{5}\right)$ & $\left(1, \frac{13}{9}, \frac{32}{9}\right)$ & $\left(\frac{25}{4}, \frac{58}{7}, 9\right)$ \\
$T$ & $\left(\frac{1}{9}, \frac{7}{58}, \frac{4}{25}\right)$ & 1 & $\left(\frac{1}{5}, \frac{1}{4}, \frac{1}{3}\right)$ & $(3,5,7)$ \\
$Q$ & $\left(\frac{9}{32}, \frac{9}{13}, 1\right)$ & $(3,4,5)$ & 1 & $(3,5,7)$ \\
$S$ & $\left(\frac{5}{34}, \frac{7}{33}, \frac{2}{5}\right)$ & $\left(\frac{1}{7}, \frac{1}{5}, \frac{1}{3}\right)$ & $\left(\frac{1}{7}, \frac{1}{5}, \frac{1}{3}\right)$ & 1 \\
\hline
\end{tabular}

Calculating fuzzy weight by formula 7 :

$(\mathrm{P}, \mathrm{T}, \mathrm{Q}, \mathrm{S})=[(1.57,2.01,2.89),(0.48,0.57,0.74),(0.75,1.32,1.77),(0.19,0.20,0.23)]$

\subsection{Calculating of each fuzzy score for each substitute program}

The rate group grades each factory by table 3 , and grades their linguistic scores of for each principle by table 4 , then gives the linguistic scores comparative numbers.

Table 4. Each linguistic score about each substitute program

\begin{tabular}{|c|c|c|c|}
\hline & $\bar{A}$ & B & $\bar{C}$ \\
\hline Price & $\begin{array}{l}\mathrm{P}=2350 / 2300=1.0217, \\
1<\mathrm{P} \leqq 1.05, \text { and defines } \\
\text { "bad" class. }\end{array}$ & $\begin{array}{l}\mathrm{P}=2480 / 2300=1.0783, \\
\mathrm{P}>1.05 \text { and defines "very } \\
\text { bad" class. }\end{array}$ & $\begin{array}{l}\mathrm{P}=2230 / 2300=0.97 \\
0.95 \leqq \mathrm{P} \leqq 1 \text { and defines } \\
\text { "average" class. }\end{array}$ \\
\hline Time & $\begin{array}{l}\mathrm{T}=14 / 12=1.16, \\
1.1<\mathrm{T} \leqq 1.2 \text {, and defines } \\
\text { "bad" class. }\end{array}$ & $\begin{array}{l}\mathrm{T}=15 / 12=1.25, \\
\mathrm{~T}>1.2, \text { and defines " very } \\
\text { bad "class. }\end{array}$ & $\begin{array}{l}\mathrm{T}=13 / 12=1.08 \\
0.9<\mathrm{T} \leqq 1.1 \text {, and defines } \\
\text { "average” class. }\end{array}$ \\
\hline Quality & $\begin{array}{l}\text { Q1. Engineering quality: : } \\
\text { Good } \\
\text { Q2.Cooperation and } \\
\text { evaluation : Good } \\
\text { Q3.Seniority : Good( have } \\
\text { run more than } 15 \text { years) } \\
\text { Q4.Scale : } \text { Good(there are } \\
\text { 6-7 people can be } \\
\text { operated) } \\
\text { Q5.coetaneous contractor } \\
\text { amount : Good(The } \\
\text { contractor amount is } \\
\text { less than ever }\end{array}$ & $\begin{array}{l}\text { Q1. Engineering quality: : } \\
\text { Good } \\
\text { Q2. Cooperation and } \\
\text { evaluation : Very Good } \\
\text { Q3. Seniority : } \underline{\text { Good( have }} \\
\text { run more than } 15 \text { years) } \\
\text { Q4. Scale : Bad(there are } \\
\text { only 1-2 people can be } \\
\text { operated) } \\
\text { Q5. coetaneous contractor } \\
\text { amount : average(The } \\
\text { contractor amount is } \\
\text { similar as usual) }\end{array}$ & $\begin{array}{l}\text { Q1. Engineering quality: : } \\
\text { Good } \\
\text { Q2. Cooperation and } \\
\text { evaluation : Very Good } \\
\text { Q3. . Seniority : Good( have } \\
\text { run more than } 16 \text { years) } \\
\text { Q4. Scale : Bad(there are } \\
\text { only 1-2 people can be } \\
\text { operated) } \\
\text { Q5. coetaneous contractor } \\
\text { amount : Good(The } \\
\text { contractor amount is less } \\
\text { than ever }\end{array}$ \\
\hline $\begin{array}{c}\text { Securely and } \\
\text { Sanitary }\end{array}$ & $\begin{array}{l}\text { S1. Securely and Sanitary } \\
\text { management situation: } \\
\text { Good } \\
\text { S2. .Cooperation and } \\
\text { evaluation : } \text { Average } \\
\text { S3. The record on previous } \\
\text { accident occurrence: } \\
\text { Bad.( because there } \\
\text { occurred one accident) }\end{array}$ & $\begin{array}{l}\text { S1. Securely and Sanitary } \\
\text { management situation:- } \\
\text { Very Good(The factory } \\
\text { demands every worker } \\
\text { to keep the site tidy and } \\
\text { neat. S2. .Cooperation } \\
\text { and evaluation : Good. } \\
\text { S3. The record on previous } \\
\text { accident occurrence:- } \\
\text { Good (because there are } \\
\text { no accidents occurred } \\
\text { before.) }\end{array}$ & $\begin{array}{l}\text { S1 Securely and Sanitary } \\
\quad \text { management situation: } \\
\quad \text { Good } \\
\text { S2. S2. . Cooperation and } \\
\quad \text { evaluation : Good. } \\
\text { S3. The record on previous } \\
\begin{array}{l}\text { accident occurrence: Good } \\
\text { (because there are no } \\
\text { accidents occurred during } 16 \\
\text { years.) }\end{array}\end{array}$ \\
\hline
\end{tabular}

Table 5. L-1、 L-2 the fuzzy score table for each hierarchy 


\begin{tabular}{c|c|c|ccccc|ccc} 
& $P$ & $T$ & $Q$ & & & & & $S$ & & \\
& & & $Q 1$ & $Q 2$ & $Q 3$ & $Q 4$ & $Q 5$ & $S 1$ & $S 2$ & $S 3$ \\
\hline$\widetilde{h}_{A}$ & $\widetilde{3}$ & $\widetilde{3}$ & $\widetilde{7}$ & $\widetilde{7}$ & $\widetilde{7}$ & $\widetilde{7}$ & $\widetilde{7}$ & $\widetilde{7}$ & $\widetilde{5}$ & $\widetilde{3}$ \\
$\widetilde{h}_{B}$ & $\widetilde{7}$ & $\widetilde{1}$ & $\widetilde{7}$ & $\widetilde{9}$ & $\widetilde{3}$ & $\widetilde{3}$ & $\widetilde{5}$ & $\widetilde{9}$ & $\widetilde{7}$ & $\widetilde{7}$ \\
$\widetilde{h}_{C}$ & $\widetilde{5}$ & $\widetilde{5}$ & $\widetilde{7}$ & $\widetilde{7}$ & $\widetilde{7}$ & $\widetilde{3}$ & $\widetilde{7}$ & $\widetilde{5}$ & $\widetilde{5}$ & $\widetilde{7}$
\end{tabular}

By table $5, \tilde{h}_{A}, \tilde{h}_{B}, \tilde{h}_{C}$ was the score of A, B and C.

\subsection{Calculating fuzzy score utilities}

\section{$\underline{\mathbf{L}-2}$}

According to weight (Q1, Q2, Q3, Q4, Q5), to grade the score of Q1 Q5 (table 5) and to calculate fuzzy utilities with Fuzzy Integral method mentioned section 3.4 in the research, so the fuzzy utilities are fuzzy scores toward "quality" principle; similarly, the fuzzy utilities of S1-S3 are fuzzy integral of "security-sanitary" principle. The fuzzy scores of Q, S as following:

Table 6. L-1 the fuzzy utility score table for each hierarchy

\section{$\underline{\mathbf{L}-1}$}

\begin{tabular}{c|cccc} 
& $P$ & $T$ & $Q$ & $S$ \\
\hline$\widetilde{h}_{A}$ & $\widetilde{3}$ & $\widetilde{3}$ & $(5.00,7.00,9.00)$ & $(2.96,5.32,7.7)$ \\
$\widetilde{h}_{B}$ & $\widetilde{7}$ & $\widetilde{1}$ & $(3.30,5.21,7.46)$ & $(5.73,7.79,9.00)$ \\
$\widetilde{h}_{C}$ & $\widetilde{5}$ & $\widetilde{5}$ & $(3.74,6.24,8.91)$ & $(3.17,5.18,7.22)$
\end{tabular}

According to weight (P,T,Q,S), to grade the score of P、T、Q、S (table 6) and to calculate fuzzy utilities of three contractors with Fuzzy Integral method.

\subsection{Result}

The fuzzy utilities of factory A, B, C was $\widetilde{u}_{A}=(1.66,3.97,6.10), \widetilde{u}_{B}=(3.01,4.93,7.78)$ and $\widetilde{u}_{C}=(3.11,5.28,7.47)$. The fuzzy utilities “ $\widetilde{u}_{A}, \widetilde{u}_{B}, \widetilde{u}_{C}$ ” expressed as fig.4.

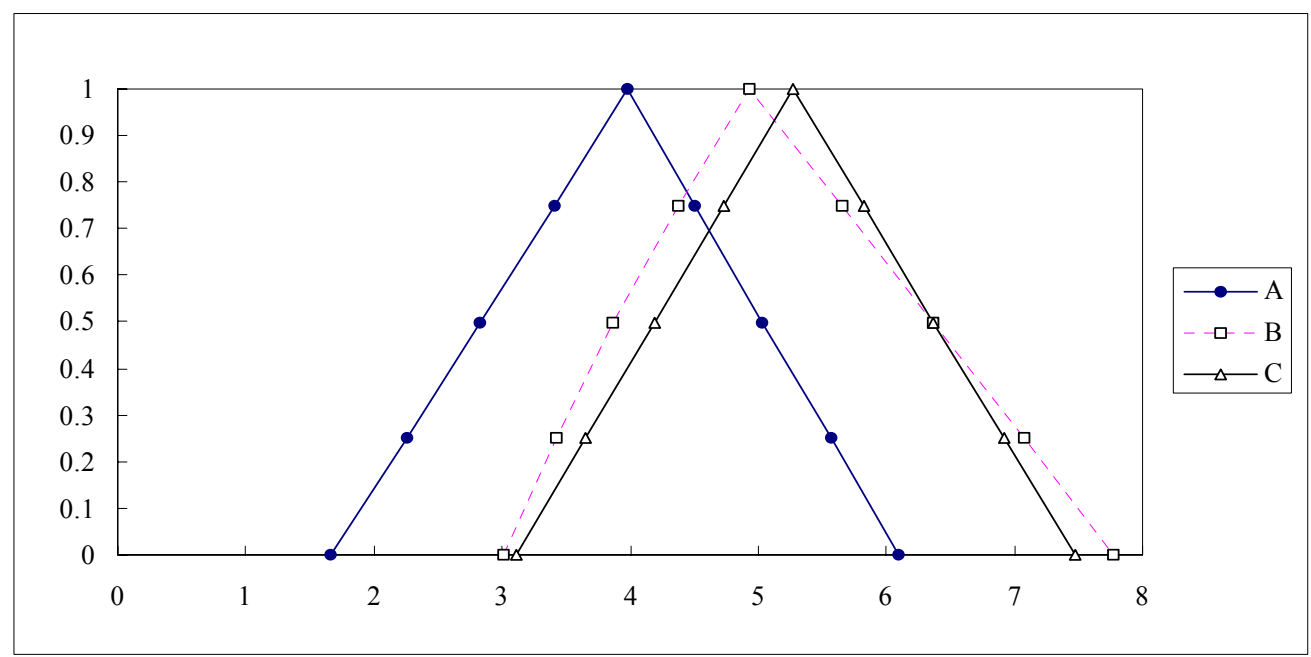

Fig.4 The membership function of fuzzy utilities " $\widetilde{u}_{A}, \widetilde{u}_{B}, \widetilde{u}_{C}$ "

Now transforms $\alpha=0 、 0.25 、 0.5 、 0.75 、 1$ into $\alpha$-cut, and the result as fig.5 and fig.6. From fig.5 and fig.6, we can know whena $>0.5$, that is, the satisfaction of higher utilities about three contractors and their order is : $\quad \mathrm{C} \rightarrow \mathrm{B} \rightarrow \mathrm{A}$. 


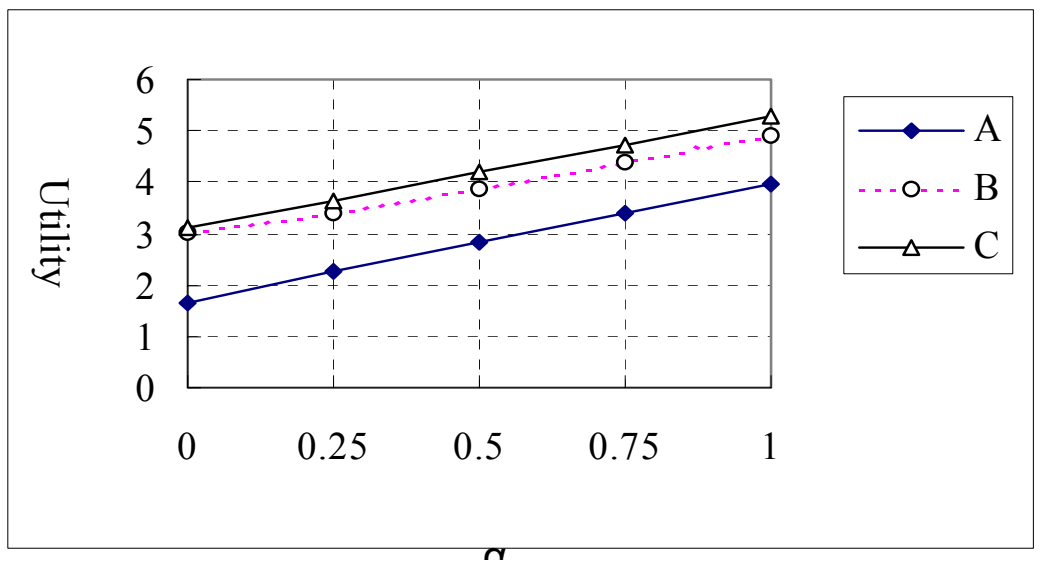

Fig.5 $\alpha$-cut(left side)

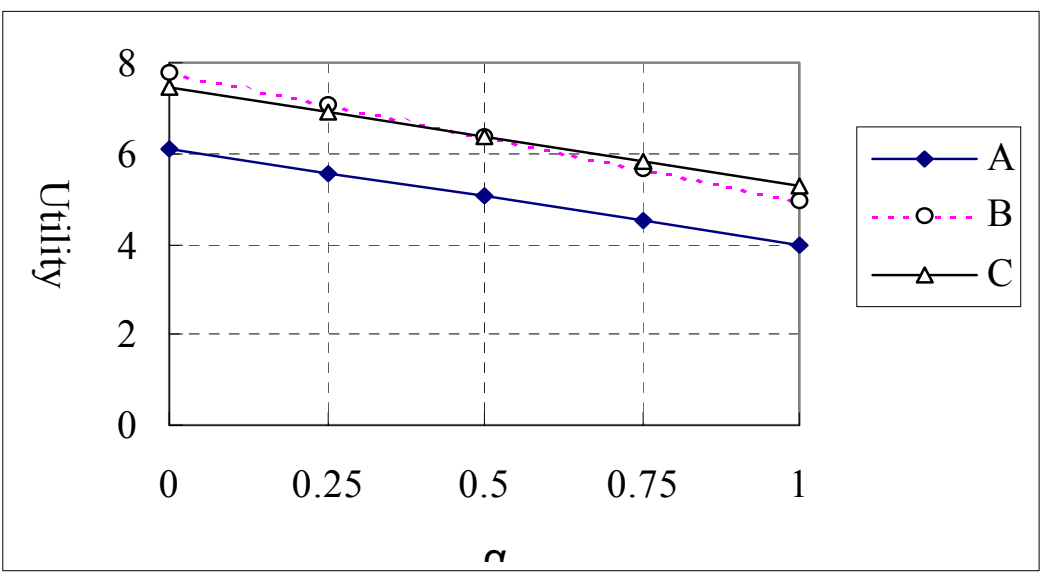

Fig.6 $\alpha$-cut(right side)

\subsection{Comparative with traditional weighted method}

From the fuzzy weight and utilities by above 4.2 and 4.3 , calculating them by formula 8 and the result are as fig.7.

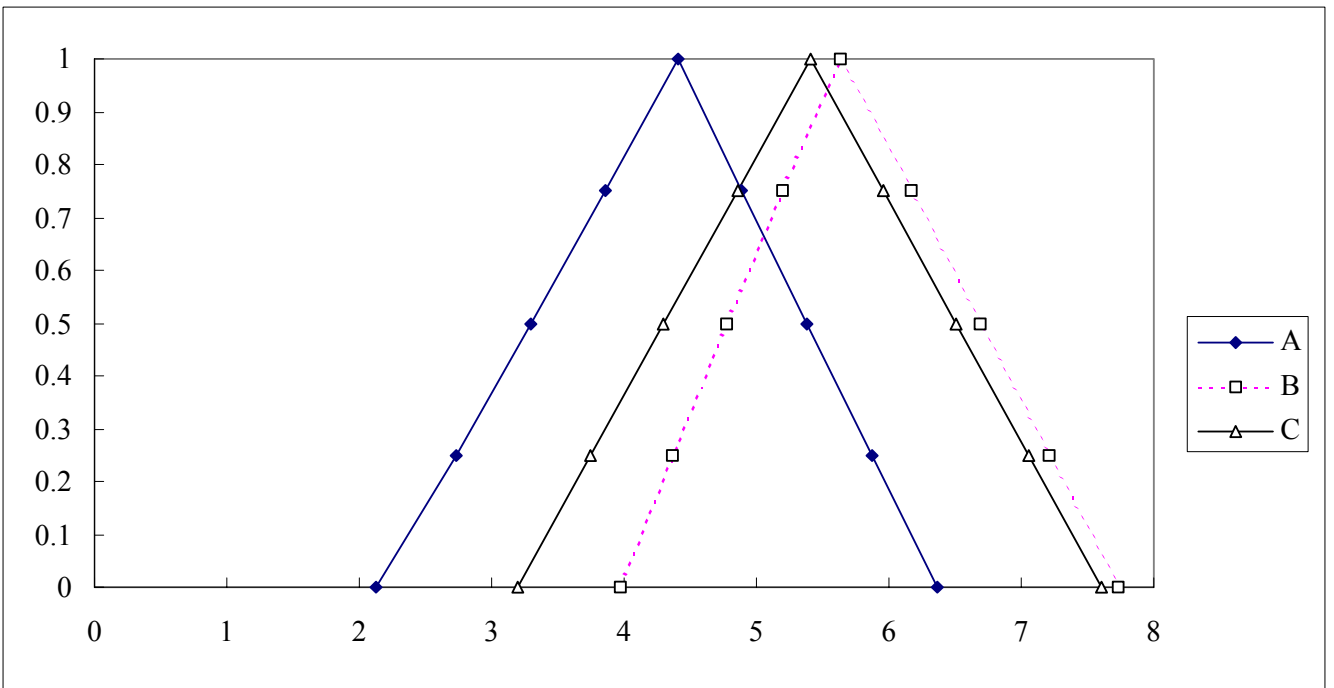

Fig.7 The membership function of traditional utilities " $\widetilde{u}_{A}, \widetilde{u}_{B}, \widetilde{u}_{C}$ "

From fig.7, we can know that just transforms any aintoa-cut, the order list are all $\mathrm{B} \rightarrow \mathrm{C} \rightarrow \mathrm{A}$, and that differentiates the results $(\mathrm{C} \rightarrow \mathrm{B} \rightarrow \mathrm{A})$ of using fuzzy integral method.

According to the scores of the three contractors, quality of contractor A is better than other contractors, it doesn't equip with any competition power on price and time, as a whole, it is a contractor which 
emphasizes quality but lakes management efficiency; as for contractor B, most cheap price, average quality, and longest time, as a whole, it is a contractor which cuts price for competition; as for contractor $\mathrm{C}$, the price, time, quality, and security-sanitary are all more than average, so it is a balanced contractor. So as to achieving multi-objective management, contractor $\mathrm{C}$ should be elected. Therefore, fuzzy integral method can considers each influence of the principle toward the non-additive weight contractor, and also can filter the situation of each principle, which taking the more for the less. On this problem, say contractor B improves the competition by cutting price, cause its dominance on price will compromise the weakness on other principles, so the total utilities is highest if uses traditional weighted method; however, the total utilities of contractor $\mathrm{C}$ whose each principle is more than average is highest by adopting fuzzy integral method. Therefore, fuzzy integral method will emphasize balanced development among each principle, and it is more realistic.

\subsection{Discussion about $\lambda$ value}

The main point about fuzzy integral is that evaluating one thing should consider the interaction among multi-factors and result in an ideal emphasis and satisfaction. Then draw out the most satisfactory factor and put the weight of the most satisfactory factor as base, then gradually add the next highest factors. After adding, it should enhance emphasis depending on $\lambda$ value. Different will affect the calculation of total utilities, and next affect ranking. For discussing the influence between $\lambda$ value and ranking, we will let fuzzy weight and fuzzy score clarify ( puta=1.0), and calculate fuzzy utilities with different values, so the result as fig.8.

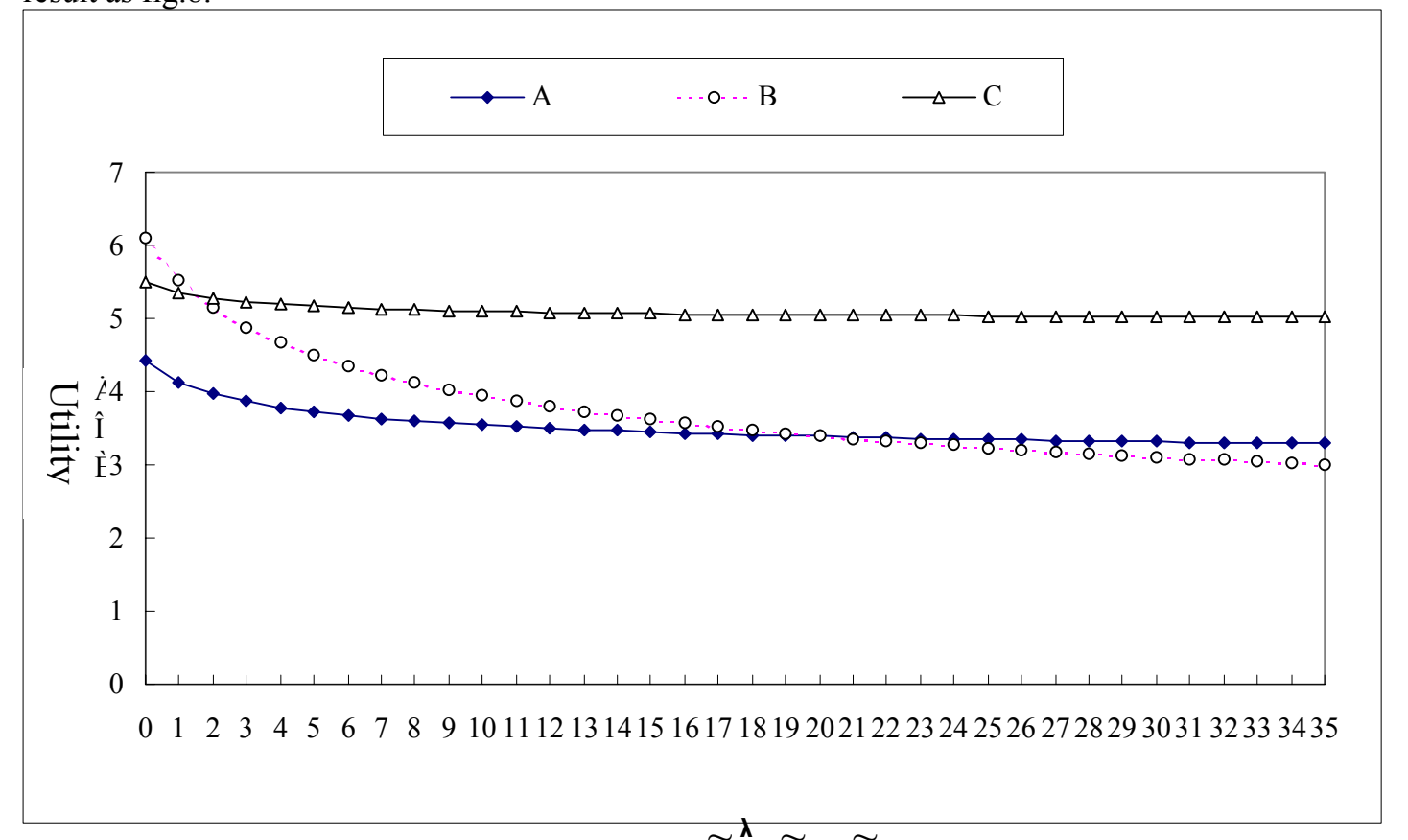

Fig.8 The inference of $\widetilde{u}_{A}^{\lambda}, \widetilde{u}_{B}, \widetilde{u}_{C}$ by conducting each $\lambda$

As fig. 8 , whena $=1.0$, its weight confirms to additive, but when there is no substitute or multiple, $\tilde{u}_{B}>\widetilde{u}_{C}>\widetilde{u}_{A}$, the ranking is $\mathrm{B} \rightarrow \mathrm{C} \rightarrow \mathrm{A}$, similar to the result of using traditional weighted method; when $2<\lambda<20$, that is, the weight confirms to additive and the multiple is common among principles, $\tilde{u}_{C}>\tilde{u}_{B}>\tilde{u}_{A} \quad$, and the ranking is $\mathrm{C} \rightarrow \mathrm{B} \rightarrow \mathrm{A}$. Hence we can know that traditional weighted method is suitable for the problems of additive weight, on the contrary, when the problems whose weight is not additive, fuzzy integral method is more appropriate, furthermore, what the problem which its weight is additive belongs to the exception to what the problem which its weight is non-additive, so the applying range of fuzzy integral is broader than that of traditional integral. When $\lambda>20$, that is, multiple is pretty high among the principles, $\tilde{u}_{C}>\widetilde{u}_{A}>\widetilde{u}_{B}$, and the ranking is $\mathrm{C} \rightarrow \mathrm{A} \rightarrow \mathrm{B}$, from fig.8, it shows that contractor B is affected most by $\lambda$, and contractor C is affected least by $\lambda$. Then we observe table6, 
differences among each principle score of contractor $\mathrm{B}$ are greatest $(\mathrm{S}$ differentiates $\mathrm{T}$ equal to 6.79), contractor $\mathrm{C}$ toward each principle are more average, near 5.That is, the bigger $\lambda$ is, the synergism each factor is, the more obvious differences of satisfaction( is equivalent to score) of each substitute program . However, the $\lambda$ value of the problem, based on the scholar, the designer, and the management three aspects, is $2<\lambda<5$, so the ranking of the problem is $\mathrm{C} \rightarrow \mathrm{B} \rightarrow \mathrm{A}$.

\section{Conclusion and Suggestion}

The research applies AHP for evaluating engineering contractor, and replaces the total addition method in fuzzy AHP method of fuzzy measurement and fuzzy integral method, and offers the fuzzy integral method whose weight is fuzzy number to omit to clarifying during evaluating process; lest the hemorrhage of data, that is, the interviewees should answer with linguistic scale, at last obtains the total fuzzy utilities of each substitute program so as to favoring the deciders to make optimal decision.

From each score of three contractors examples, contractor A focuses quality but lakes of management efficiency; contractor B cuts price for the competition; contractor C emphasizes balanced development. To achieve the objective mentioned on constructing management, contractor $\mathrm{C}$ is more appropriate and confirms to multi-objectives. Even if the same weight and score, the ranking of traditional weighted method is $\mathrm{B} \rightarrow \mathrm{C} \rightarrow \mathrm{A}$, but the ranking of fuzzy integral method is $\mathrm{C} \rightarrow \mathrm{B} \rightarrow \mathrm{A}$, that is, using fuzzy integral method for choosing optimal contractor is more realistic.

Among three contractors, contractor B obtains the most difference on each principle ( $\mathrm{S}$ differentiates $\mathrm{T}$ equal to 6.79), and contractor $C$ gets more average scores, 5 or so. However, the results prove that when $\lambda$ $>20$, the ranking of contractor $\mathrm{C}$ are the same, that of contractor $\mathrm{B}$ backs off, therefore the more $\lambda$ value and the higher the multiple are, the more obvious difference of satisfaction (is equivalent to score) of each substitute program. When $\lambda$ value is 0 , the results of fuzzy integral and the traditional weighted method are the same, that is, when $\lambda$ value is 0 , traditional weighted is the exception, therefore, traditional weighted method is suitable for the problem whose weight is additive, but fuzzy integral method is more appropriate for solving the problem whose weight is non-additive and the exception of the weight confirms to additive, in conclusion, the apply range is more broad than traditional weighted method.

The evaluating principle in the research could evaluate in pretty aspects, such as single engineering, but in real application, the research suggests that choosing different evaluating principle with fuzzy AHP method to calculating respect weight (i.e. mold engineering is fixed weight, cementation engineering is fixed weight, then develop the professional system to calculating comprehensive evaluating for each contractors as the references when deciding.

Furthermore, the research does not investigate on $\lambda$ value and the differences and comparativeness among each principle, besides research on damages all traditional principles to rearranging and reclassifying, and those directions will be good direction in the future; moreover, the pair comparative matrix many professionals offered is the most easy geometric mean method, but the method is might too simple to keep the whole data, in conclusion, the future research can improve on the Group decision making the evaluating method offered in the research and provide the reference for deciders.

\section{Reference}

Buckley, J.J. (1985), "Fuzzy hierar chical analysis”, Fuzzy Sets and Systems 17, pp.233-247.

Cheng, C.H. and Mon, D.L (1994), "Evaluatiog weapon system by analytical hierarchy process based on fuzzy scales", Fuzzy Sets and Systems 63, pp.1-10.

Chen, Ting-Yu; Wang, Jih-Chang; and Tzeng, Gwo-Hshiung, "Identification of General Fuzzy Measures by Genetic Algorithms Based on Partial Information", IEEE Transactions on Systems, Man, and Cybernetics 
Part B: Cybernetics, Vol. 30B, No. 4, pp. 517-528 2000. SCI

Grabisch, M. (1995), "Fuzzy integral in multicriteria decision making", Fuzzy Sets and Systems 69, pp.279-298.

Ghotb, F. and Warren, L. (1995), “A case study comparision of the Analytic Hierarchy Process and a fuzzy decision methodolgy", The Engineering Economist, Spring, Vol.40, No.3, pp.233-245.

Kerzner,H. (1989), Project management: a system approach to planning scheduling and controlling, New York: Van Nostrand Reinhold, pp.759-764 .

Klir, G.J. and Yuan, B. (1995), Fuzzy Sets and Fuzzy Logic, Prentice-Hall International, Inc.

Laarhoven, P.J.M and Pedrycz, W. (1983), “A fuzzy extension of Saaty“s priority theory”, Fuzzy Sets and Systems 11, pp.229-241.

Nakajima, N., Takeda, E. and Ishii, H. (1994), Fuzzy Theory for Social Science, Shokabo, Tokyo.

Saaty, T.L. (1977), "A scaling method for priorities in hierarchical structures, Journal of Mathematical psychology 15, pp.234-281.

Saaty, T.L. (1980), The Analytic Hierarchy Process, New York, Mcgraw-Hill.

Tzeng, G.H. and Teng, J.H. (1993), "Transportation investment project selection with fuzzy multiobjectives", Transportation planning and Technology 17, pp.91-112.

Chen, Y. W. and Tzeng, G. H. (1999), "Using Fuzzy Integral for Evaluating Subjectively Perceived Travel Costs in a Traffic Assignment Model," European Journal of Operational Research. (EOR 4212A Theory and Methodology, Pap.No.: 99213, Accepted: 99-11-04). SCI, SSCI (forthcoming) 